\title{
Multifaceted Usage of HPV Related Tests and Products in the Management of Cervical Cancer - a Review
}

\author{
Sivalingam Nalliah¹, Barani Karikalan ${ }^{2 *}$, Kumaraswamy Kademane ${ }^{2}$
}

\begin{abstract}
HPV viruses are integral to the development of cervical cancer. The pathogenesis has been extensively studied. To date, numerous HPV tests and products have been developed and successfully utilized in diagnosis, treatment and prevention of cervical cancer. The HPV DNA test, when combined with other routine cervical cancer screening and diagnostic tests namely exfoliative cytology, visual inspection with acetic acid (VIA) and colposcopy has increased the detection rate of cervical cancer. HPV DNA products could also be measured in other body fluids like urine, lymph node tissue, and serum. HPV association could also be quantified by measuring other parameters like HPV mRNA, viral load, viral integration and methylation status. Vaccination against HPV has been found to decrease the incidence of cervical cancer. Further, therapeutic vaccines for cervical cancer against HPV continue to evolve. All these findings pertaining to HPV could possibly decrease the incidence of cervical cancer in the near future. This review aims to give an overview of the HPV tests and products in use and those under trial currently.
\end{abstract}

Keywords: HPV - DNA test - triage - cervical intraepithelial lesion - cervical cancer

Asian Pac J Cancer Prev, 16 (6), 2145-2150

\section{Introduction}

HPV are a group of heterogeneous DNA viruses which infect the keratinocytes of human skin and mucosa to cause a variety of lesions like skin warts, genital warts, papilloma, cervical intraepithelial lesion and cancer. The virus is transmitted by direct contact, sexual contact and is transmitted to the newborn from the infected mother via vertical transmission. Recently, HPV have been studied extensively though knowledge of HPV associated lesions has been known since ancient times. Hippocrates described HPV in the $4^{\text {th }}$ century as an ulcer transforming into cervical cancer left untreated. Thereafter, in the19th century, an Italian surgeon Antonio Domenico RigoniStern inferred development of cervical cancer from an infection. George N. Papanicolaou, a Greek scientist, started describing virus related changes in squamous cells. His findings emerged into the Pap smear screening test later. Strauss, in 1949 and Crawford, in 1963 respectively provided electron microscopic structure and physical properties of the virus. Harald Zur Hausen, the Father of Virology, first proposed the relationship between HPV and cervical cancer in 1970. Now HPV is the most elaborately studied virus. Apart from cervical cancer, the virus has been known to be a cause for many other non-genital cancers (Ioannis et al., 2014).

After the establishment of the role of HPV virus in the causation of cervical cancer, the concept has been extensively used in diagnosis, prevention and treatment of the cancer. The objective of this review is to give a short overview of different HPV related tests and products used in the management of HPV associated cervical cancer.

\section{The HPV virus}

The HPV virus is a small, 8-kilo base double stranded DNA virus belonging to the Papillomaviridae family. There are more than 100 different types of HPV virus causing proliferative lesions in human skin and mucosal surfaces. Not all lesions proceed to carcinoma. About $60 \%$ of low grade squamous intraepithelial lesions regress spontaneously. In the presence of co-factors like genetic factors, multiparity, oral contraceptive pill use, cigarette smoking, immunodeficiency and other cervical infections like Herpes simplex and Chlamydia; $15 \%$ of the low-grade intraepithelial lesions proceed to highgrade lesions. Thirty to seventy per cent of these highgrade intraepithelial lesions were found to evolve into invasive cancers over a duration of ten years. HPV virus is detected in $96.6 \%$ of all the cervical cancers (Faruk et al., 2014). The most common types of HPV are HPV 16, $18,58,33,45,31,52,35,59,39,51$ and 56 in the order of decreasing frequency. Seventy to seventy six percent of all cervical cancers worldwide are associated with HPV16/18 except Asia where the association is 68-82\% (Li et al., 2010). Apart from being implicated in squamous 
cell lesions, HPV viruses are also associated with cervical adenocarcinomas especially HPV18. Eighty nine percent of cervical adenocarcinomas are associated with high risk HPV infection, out of which $54 \%$ are of type 18 (Baalbergen et al., 2012).

\section{The HPV DNA test}

The presence of HPV DNA in the cervix is detected by two efficient methods i.e. hybrid capture II assay and polymerase chain reaction. These tests are used to detect the presence of high-risk HPV viral infections that are associated with cancer generation. A negative test indicates absence of high risk HPV viral infection. The routine cervical cytology test used for screening cervical cancer has a very low sensitivity. Numerous trials have been conducted to demonstrate sensitivity, specificity and detection rates of HPV DNA. In a comparative study conducted by Shalini et al. (2002) where 4075 women participated, sensitivity and specificity of thin layer Pap smear and HPV DNA test were compared. Both tests were done simultaneously on all participants. Patients with positive test were referred for colposcopic biopsy. Patients diagnosed with Atypical Squamous Cells of Undetermined Significance (ASCUS) and any higher in Pap smear or presence of high risk HPV DNA by hybridization capture method/polymerase chain reaction method were considered positive. The prevalence of Cervical Intraepithelial Neoplasia (CIN)3 or any higher lesion was $3.2 \%$. The sensitivity of HPV PCR $(88.2 \%)$ and HC assay (90.8\%) was higher than thin layer pap smear (61.3\%) for identifying CIN3 or any higher lesion. But specificity of thin layer Pap smear $(82.4 \%)$ was higher than PCR test (78.8\%) and HC assay (72.6\%) in detecting CIN3 and higher lesions. The study concluded that HPV DNA testing could be used as a screening test for cervical cancer. Ogilvie et al. (2012) did an RCT trial comparing the efficacy of CIN2 detection in ASCUS patients by liquid based cytology and HPV DNA test. Use of HPV DNA test as a potential screening tool was apparent. Othman and Hayati Othman (2014), in their comparative study conducted in Malaysia found increased presence of hrHPV in Pap smears with abnormal cytological findings. They suggested strategizing and implementing HPV DNA test only in cases with ambiguous cytology might improve the cost effectiveness of cervical cancer screening programs in developing nations like Malaysia.

\section{HPV DNA and colposcopy}

The HPV DNA test as a screening test could be used to detect more CIN lesions without increasing the colposcopy referral rate. The HART multicentric screening study involved 11083 women older than 30years, with borderline cytological changes in Pap smear. Women with positive high risk HPV test considered positive (825) were randomly divided into two groups. One group was subjected to immediate colposcopy and the other underwent repeat HPV and cytology testing followed by colposcopy after 12 months. The authors concluded that both the methods were found to be equally effective. The positive women in second group who got negative results during repeat testing at 12 months showed no CIN lesion on colposcopy. Hence, it was inferred that patients with high-risk positive HPV or borderline cytological changes can be followed up for up to 12 months with repeat testing. By doing so, detection rate of CIN lesions could be improved without increasing referral for colposcopy (Cuzick et al., 2003).

HPV DNA test done along with routine Pap smear could reduce colposcopy referral rate in ASCUS patients. Pap smears showing ASCUS may be from CIN2, 3 or invasive cervical cancer. The recommended options for any ASCUS case would be immediate colposcopy, cytology follow up or HPV DNA testing (ASCUS-LSIL Triage Study (ALTS) Group, 2003). A retrospective analysis done on 594 patients with ASCUS who had undergone either immediate colposcopy or colposcopy if HPV positive showed that the detection rate of CIN was higher with colposcopy referred after positive HPV test than with immediate colposcopy (Kececioglu et al., 2013).

\section{HPV DNA and visual inspection with acetic acid}

Visual inspection with acetic acid (VIA) is an inexpensive alternative to cytology for regions with low resources. It gives rapid results and needs minimally trained staff. But VIA done alone is not sufficient to diagnose cervical lesions. But HPV DNA testing done along with VIA might provide a good outcome. Jeronimoa et al. (2003) conducted a population-based study of cervical neoplasia in Costa Rica involving 5564 women belonging to 25-69 years age group. Subjects were followed up with varying tests like cytology, cervicogram, clinical examination, HPV DNA test and finally referred to colposcopy. The cervicograms of women with positive HPV DNA tests were evaluated to simulate VIA. HPV test and VIA done together gave a sensitivity of $65.5 \%$ and specificity of $96.9 \%$. HPV testing done alone gave a higher sensitivity of $81.0 \%$ and a lower specificity of $90.8 \%$. Thus, VIA when combined with HPV test could provide better results than cytology especially if a low cost, less time consuming HPV test was provided. Following this method could also be very useful to detect cervical lesions in resource poor areas where cervical cancer screening cannot be done very often. Pimple and Shastri (2014), in a cross sectional cervical cancer study involving 3625 women found that VIA increased the sensitivity and specificity of HPV DNA test in detecting CIN2 lesions. They proved that VIA followed by HPV DNA test could be a cost effective cervical cancer screening strategy in low resource areas.

\section{HPV in the urine}

Most women especially in low socio-economic group consider undergoing cervical cancer screening embarrassing and many consider intravaginal examination with a vaginal speculum painful. These factors can contribute to patients' reluctance to subject themselves to screening programs contributing to increasing cancer statistics (Baskaran et al., 2013). So other non-invasive 
methods of HPV detection, if available would be appreciable approaches that could increase efficiency of cervical cancer screening. Detecting HPV DNA in urine samples could be a convenient and alternative method for routine invasive method of HPV detection. Majid et al (2014) studied 1000 women; HPV detection was done by PCR method in urine and cervical samples. An $80 \%$ concordance was noted. Further in urine samples, the sensitivity for detection of high-grade squamous intraepithelial lesion was $100 \%$ with a specificity of $80 \%$. Thus, HPV detection in urine could be a useful alternative screening method. Enerly et al (2013) suggest the use of urine samples in adolescents to study vaccine impact in a large scale. In their review article, where they analyzed about 14 studies that measured HPV in paired urine and cervical samples, they found $75-100 \%$ concordance rate between urine and cervical samples in HPV detection.

\section{HPV in lymph nodes}

The presence of metastatic deposits in lymph nodes is an important prognostic factor in cervical cancer. The risks of recurrence even in non-metastatic lesions reach up to $15 \%$. A systematic review done on all available data about HPV DNA detection in pelvic lymph nodes in cervical cancer patients found that there was a high correlation between positive HPV DNA and metastasis in pelvic lymph nodes which lead to a hypothesis that HPV DNA detection in pelvic lymph nodes could be a risk factor for recurrence and poor prognosis in cervical cancer (Marco Noventa et al., 2014).

\section{Circulating HPV DNA}

HPV DNA integration into the genome of carcinoma cells is a highly specific molecular marker of tumor DNA. Circulating DNA detected using the specific molecular motif is a newly emerging marker, which could be used as prognostic factor for cervical cancers. It could also be used to detect minimal residual disease and recurrence. Circulating DNA might be released more easily from recurrent lesions and metastatic lesions than from primary lesion (Widschwendter et al., 2003). So quantitative estimation of circulating DNA might be used to followup the course of the disease. This is supported by a study conducted by Campitelli et al. (2012) on 16 patients with cervical cancer associated with HPV16/18 and for which viral integration motif was already known. Circulating serum DNA for all the patients was analyzed. Out of 13 patients with tumor size more than $20 \mathrm{~mm}, 11$ patients had circulating tumor DNA. Sequential analyses of circulating DNA in two patients showed that concentration of the circulating DNA was related to the tumor dynamics.

\section{HPV DNA and micronuclei}

Micronucleus is produced when a chromosome segment fails to incorporate into the nucleus during cell division. It can be readily identified under light microscopy representing genetic damage seen in tissue exposed to carcinogens even before clinical symptoms arise. Since HPV is known to cause cytological changes in cervical cells, it is hypothesized that micronucleus may be associated with the presence of HPV DNA (LealGarza et al., 2002). Cassel et al. (2014) investigated the hypothesis by analyzing 158 cytological smears. Genome amplification of HPV DNA was done for highrisk types 16,18,31,33,39,45 and 59. Micronucleus was detected in 11 out of 20 HPV DNA positive samples and 36 out of 138 HPV DNA negative samples. Presence of micronucleus was higher in HPV positive samples. Also, absence of micronuclei in HPV negative samples may be used to rule out presence of HPV. Thus it was concluded that micronucleus could be used to predict the presence or absence of HPV DNA. Gutierrez et al. (2010) had previously also demonstrated an association between HPV infection and increased frequency of micronuclei in a hospital based case control study.

\section{HPV mRNA}

Trials are being done to detect whether HPV mRNA could be used as a better biomarker for cervical cancers. Persson et al. (2014) did a comparative study to compare the efficacy of HPV DNA and HPV mRNA in detecting cervical lesions. Two hundred and nineteen women who were cytologically diagnosed with ASCUS and low grade squamous intraepithelial lesion (LSIL) along with HR HPV positivity involved in a population based cervical cancer-screening program in Stockholm, Sweden were included. HPV mRNA test was done by APTIMA (GeneProbe Inc., San Diego, CA, USA). The women were followed up for 4 years. During the follow up, $36 \%$ of women developed CIN2 or higher lesion. HPV mRNA test was more sensitive (78-100\%) in detecting CIN2 or higher lesion but less specific $(50 \%)$. Thus, they reported that HPV mRNA could be used as a triage test for women with ASCUS but women with LSIL might require more focused tests.

Castro et al. (2013) conducted another trial to test the efficacy of HPV mRNA test and also to determine if it can be used as to triage HPV DNA test. The study included 165 women with HPV DNA positive results. HPV mRNA test (NucliSENS-EasyQ HPV E6/E7-mRNA-assay Biomerieux) was done on all samples. Women who had cytological abnormalities were referred for colposcopy. $25.8 \%$ of the women with ASCUS or LSIL and $89.8 \%$ of women with High grade squamous intraepithelial lesion (HSIL) had CIN2 or higher lesion. The sensitivity of mRNA in detecting CIN2 or higher lesion was $81-84 \%$ and specificity was $52-80 \%$. Further, mRNA reduced the number of colposcopies done. Women without cytological abnormalities were followed up for 18 months, 34\% of which developed CIN2 or higher lesion. mRNA sensitivity and specificity in detecting CIN2 or higher lesion were $83.3 \%$ and $89.6 \%$ respectively, proving that the mRNA test could be used to triage HPV DNA test. Besides, HPV mRNA test could be used to manage HPV DNA positive women who are colposcopy and histology negative. Rossi et al. (2013) evaluated 673 women with negative colposcopy and positive HPV DNA. They were followed up for 25 months. Seven developed CIN2 lesions. 
HPV mRNA test was done earlier or on all the participants. Out of the 7 CIN2 cases diagnosed, 3 were from $82 \mathrm{HPV}$ mRNA positive women, 2 were from $250 \mathrm{HPV}$ mRNA negative women and 2 were from 12 women with invalid mRNA test. This study concluded that HPV mRNA could be a good prognostic marker in the management of HPV DNA positive cases with negative colposcopy and histology. In particular, it could help to reduce the intensity of management in negative patients.

\section{HPV viral load}

The HPV DNA test is usually very sensitive but less specific and has a very low positive predictive value (Mayrand et al., 2007). To fill in this gap, quantitative HPV DNA test (viral load) could be used. Studies have been conducted to detect the correlation between viral load and cervical lesions. Schmitt et al (2013) studied viral load of 999 samples with cytological abnormalities i.e. ASCUS, LSIL and HSIL using quantitative real time PCR. After a follow up of 36 months, 79 CIN2 or higher lesions were detected on colposcopic biopsy. Viral load of samples with LSIL and HSIL were higher than other samples with negative cytological results but the difference in mean viral load between the LSIL and HSIL was not significant. The sensitivity for detecting CIN3 was $100 \%$ and specificity increased to $95.7 \%$. Thus, viral load testing was found to reduce false positive results and increase specificity and positive predictive value in detecting cervical lesions. Bencomo-Alverez et al (2012), in their study involving 46 Mexican patients with cervical lesions of various stages also found higher viral load in patients with cervical lesions than in control samples without any cervical lesion. They proposed that viral load could be a potential biomarker for predicting cervical lesions.

\section{HPV viral integration}

In cervical cancers associated with HPV, the viral DNA is integrated with the human DNA or it could be seen separately as an episome or both (Woodman et al., 2007). Studies were conducted to detect whether the physical status of the virus could be a prognostic marker for cervical cancer. Das et al (2012) reviewed 270 cervical cancer patients where HPV association was found to be $95 \%$. Amplification of Papillomavirus Oncogene Transcripts (APOT) assay was done to identify viral integration site. Viral integration was noticed in $79 \%$ of the cases. Integration preference was frequently found in chromosome loci 1p, 3q, 6q, 11q, 13q and 20q. This study conclusively implies that physical status of HPV viral DNA could be used as a prognostic marker for cervical cancer.

Viral integration occurs at different sites and is randomly distributed all over the host genome. The integration site differs with changing viral structure and the targeted host genome loci (Wentzensen et al., 2004). These integration sites could also be used as biomarker for cancer prognosis. On examination of 57 cervical cancer samples, Xu et al (2013) identified 75 viral integration sites and 39 viral-cellular DNA junctions were identified.
The novel method developed to detect integration sites includes DNA fragmentation and adaptor tagging, PCR enrichment, sequencing, data processing and validation of integration site by junction PCR. The integration sites and junctions were found to have preferential distribution in the host genome contributing to insertional mutation. Thus the developed multiplex strategy method for detection of viral integration site might be very useful and the mapped integration sites might be used as biomarker in the management of cervical cancer.

\section{HPV DNA methylation}

Methylated cytosines (meCpG) disrupt transcription factors and also change chromatin configuration. Methylation of CpG sites of HPV DNA plays an important role in carcinogenesis of cervical cancers (Kalantari et al., 2004). Quantifying the amount of methylation might help in the prediction of prognosis of cervical cancer. In a population based case control study that included 273 cervical samples by Mirabello et al (2013), 109 cases were HPV DNA positive with CIN2 lesion. When methylation at $66 \mathrm{CpG}$ sites of HPV genome was quantified in these cases by pyrosequencing assay, $88 \%$ of the $\mathrm{CpG}$ sites had higher methylation levels. Seventeen per cent of $\mathrm{CpG}$ sites with higher methylation levels in non-CIN cases developed CIN2 lesions over a follow up of 3 years. These findings point to the fact that high levels of methylation at CpG sites in HPV genome might be useful in diagnosis as well as to predict future CIN2 cases. Vasiljevic et al (2014) identified 6 genes, methylation of which could be used to detect cervical lesions. Measuring these methylated genes could be a better triage test for HPV DNA test by increasing its specificity.

\section{HPV DNA methylation panel}

Many studies have been done to identify methylation frequencies in cervical cancer genes (Wentzensen et al., 2008). If a panel of these methylation targets were identified, they could be used to distinguish lesions that progress to carcinomas and lesions that are destined to regress. Patel et al (2012) used 151 samples and identified a panel of methylation targets. Frequent methylation sites in candidate genes like ESR1, DCC, p16 and LINE1 were chosen to measure methylation levels. Methylation levels were also measured in $\mathrm{CpG}$ sites in E6 promotor region and L1 region in HPV genome in $89 \mathrm{HPV}$ positive cases. Lower methylation levels in HPV genome and higher methylation levels in candidate genes were found to be associated with abnormal cytology. Eijsink et al (2011) identified a four gene methylation panel (JAM3, EPB41L3, TERT and C13ORF18) which when combined with HPV DNA test, increased the detection rate of cervical lesions, thereby proving the efficacy of their methylation panel as a triage test for HPV DNA test.

\section{HPV prophylactic vaccine}

Two types of HPV prophylactic vaccines are currently available for protection against high-risk HPV infection 
Table 1. Summary of HPV Tests, Parameters and their Implications

\begin{tabular}{lll}
\hline Tests & & Implications \\
\hline HPV triage & HPV test before colposcopy & Reduces colposcopy referral rate Increase detection of CIN \\
& HPV test after VIA & Cost effective Increases detection rate of CIN \\
Alternate HPV detection & Urine & $75-100 \%$ concordance rate with cervical samples \\
& LN & Prognostic factor for cervical cancer \\
& Serum & Molecular marker for prognosis of cervical cancer \\
& Micronuleus in cytology & Predicts HPV infection \\
Other HPV parameters & mRNA & Triage to HPV DNA test \\
& Viral load & Increases specificity of HPV DNA test Predicts cervical lesion \\
& Viral integration & Biomarker for prognosis of cervical cancer \\
& DNA methylation & Increases specificity of HPV DNA test Predicts cervical lesion \\
\hline
\end{tabular}

i.e. quadrivalent HPV vaccine against HPV types 6,11 , 16 and 18 and bivalent HPV vaccine against types 18 and 16. Both the vaccines contain L1 protein for antigenicity in the form of virus like particle (VLP) and an adjuvant. The development of VLP in 1991 was a major break through in the development of HPV vaccines. VLP has a virus like structure made of capsomers comprising L1 proteins (Kim et al., 2014). Several studies have been conducted to test the efficacy, safety and immunogenicity of both the vaccines. Harper et al (2004) conducted a trial to test the efficacy of the bivalent vaccine; the efficacy against persistent HPV infection was found to be $95 \%$ and efficacy against cytological abnormalities associated with HPV infection was 92\% (Harper et al., 2004). Markowitz et al (2013) comprehensively reviewed vaccine efficacy of HPV quadrivalent vaccine in the United States by analyzing the prevalence of HPV infection in the vaccination and pre-vaccination era. The prevalence of HPV infection was found to be low in the vaccination era and vaccine efficacy was found to be $82 \%$.

\section{HPV therapeutic vaccine}

It is not only sufficient to prevent HPV infection, owing to the strong association of HPV infection and cervical cancers but is also necessary to discover ways to treat HPV induced cervical lesions. Many immune therapeutic and targeted therapeutic products are under development (Trimble and Frazer, 2009). Live vector vaccines, peptide vaccines, protein vaccines, DNA vaccines and dendritic cell vaccines are some of the therapeutic vaccines currently under clinical trials. Protease inhibitors, histone deacetylase inhibitors, sirna against E6/E7 proteins are some of the other products under clinical trial targeted against HPV viral products (Knoff et al., 2014).

\section{Conclusions}

Current strategies to reduce the global prevalence of carcinoma of the cervix are based on extensive research, which has conclusively linked HPV infection with the aetiopathogenesis of the disease. Social, cultural and resources for both screening and detection have strong implications. Molecular biology has made great inroads in development of predictive markers and prediction of progress of pre-malignant lesions of the cervix.

Clearly this link between HPV virus and cervical cancer has paved the way for newer strategies in prevention and management of pre-invasive and invasive cervical cancers. In this review, various parameters and products related to HPV are analyzed and presented. All these tests should be considered in designing newer strategies in cervical cancer screening programs for detection of early lesions in a cost effective way.

\section{References}

ASCUS-LSIL Traige Study (ALTS) Group (2003). Results of a randomized trial on the management of cytology interpretations of atypical squamous cells of undetermined significance. Am J Obstet Gynecol, 188, 1383-2.

Baalbergen A, Smedts F, Ewing P, Snijders P, Meijer C, Helmerhorst T (2012). HPV-type has no impact on survival of patients with adenocarcinoma of the uterine cervix. Gynecol Oncol, 128, 530-4.

Baskaran P, Subramanian P, Rahman R, et al (2013). Perceived susceptibility, and cervical cancer screening benefits and barriers in Malaysian women visiting outpatient clinics. Asian Pac J Cancer Prev, 14, 7693-9.

Bencomo-Alvarez A, Limones-Perches I, Suarez-Rincon A, et al (2012). Human papillomavirus viral load in cervical intraepithelial neoplasia as a prognostic factor in a mexican population. Genetics Molecular Res, 11, 4720-7.

Campitelli M, Jeannot E, Peter M, et al (2012). Human papillomavirus mutational insertion: Specific marker of circulating tumor DNA in cervical cancer patients. PLOS One, 7, 1-5.

Cassel A, Barcellos R, Silva C, Almeida S, Rossetti M (2014). Association between human papillomavirus (HPV) DNA and micronuclei in normal cervical cytology. Genet $\mathrm{Mol}$ Biol, 37, 360-3.

Castro S, Fernandez A, Gonzalez M, TeresaSaranDiez M, CidLama A, AlvarezMartin M, et al (2013). Human papillomavirus (HPV) E6/E7 mRNA as a triage test after detection of HPV 16 and HPV 18 DNA. J Med Virol, 85, 1063-8.

Cortes-Gutierrez E, Davila-Rodriguez M, Vargas-Villarreal J, Hernandez-Garza F, Cerda-Flores R (2010). Association between human papilloma virus-type infections with micronuclei frequencies. Prague Medical Report, 111, 35-1.

Cuzick J, Szarewski A, Cubie H, et al (2003). Management of women who test positive for high-risk types of human papillomavirus: The HART study. Lancet, 362, 1871-6.

Das P, Thomas A, Mahantshetty U, Shrivastava S, Deodhar K (2012). HPV genotyping and site of viral integration in cervical cancers in indian women. PLOS ONE, 7, 41012.

Eijsink J, Lendvai A, Deregowski V, et al (2012). A four-gene methylation marker panel as triage test in high- risk human papillomavirus positive patients. Int J Cancer, 130, 1861-9.

Enerly E, Olofsson C, Nygård M (2013). Monitoring human 
papillomavirus prevalence in urine samples: A review. Clin Epidemiol, 5, 67-9.

Faruk M, Murat M (2014). Cervical premalignant lesions and their management. J Turk Ger Gynecol Assoc, 15, 109-1.

Harper D, Franco E, Wheeler C, Ferris D, Jenkins D, Schuind A, et al (2004). Efficacy of a bivalent L1 virus-like particle vaccine in prevention of infection with human papillomavirus types 16 and 18 in young women: A randomised controlled trial. Lancet, 364, 1757-5.

Ioannis N, Demetrios GS (2014). The paediatric story of human papillomavirus (review). Oncology Letters, 8, 502-6.

Jeronimoa J, Castlea P, Herrerob R, Burkc R, Schiffmana M (2003). HPV testing and visual inspection for cervical cancer screening in resource-poor regions. Int J Gynecol Obstet, 83, 311-3.

Kalantari M, Calleja-Macias I, Tewari D, et al (2004). Conserved methylation patterns of human papillomavirus type $16 \mathrm{DNA}$ in asymptomatic infection and cervical neoplasia.J Virology, 78, 12762-2.

Kececioglu M, Seckin B, Baser E, et al (2013). Cost and effectiveness comparison of immediate colposcopy versus human papillomavirus DNA testing in management of atypical squamous cells of undetermined significance in turkish women. Asian Pac J Cancer Prev, 14, 511-4.

Kim K, Park S, Ko K, Yi S, Cho Y (2014). Current status of human papillomavirus vaccines. Clin Exp Vaccine Res, 3 , 168-5.

Knoff J, Yang B, Hung C, Wu T (2014). Cervical cancer: Development of targeted therapies beyond molecular pathogenesis. Curr Obstet Gynecol Rep, 3, 18-2.

Leal-Garza C, Cerda-Flores R, Leal-Elizondo E, CortesGutierrez E (2002). Micronuclei in cervical smears and peripheral blood lymphocytes from women with and without cervical uterine cancer. Mutation Res, 515, 57-2.

Li N, Silvia Franceschi S, Howell-Jones R, Snijders P, Clifford G (2010). Human papillomavirus type distribution in 30,848 invasive cervical cancers worldwide: Variation by geographical region, histological type and year of publication. Int J Cancer, 128, 927-5.

Majid S, Teng S, Malik I, et al (2014). Estimation of human papilloma virus DNA in urine specimens as a marker for cervical cancer at an early stage. Int J Curr Microbiol App Sci, 3, 698-1.

Marco Noventa M,Ancona E, et al (2014). Usefulness, methods and rationale of lymph nodes HPV-DNA investigation in estimating risk of early stage cervical cancer recurrence: A systematic literature review. Clin Experimental Metastasis, 31, 853-7.

Markowitz L, Hariri S, Lin C, et al (2013). Reduction in human papillomavirus (HPV) prevalence among young women following HPV vaccine introduction in the united states, national health and nutrition examination surveys, 2003-2010. J Infect Disease, 208, 385-3.

Mayrand M, Duarte-Franco E, Rodrigues I, et al (2007). Human papillomavirus DNA versus papanicolaou screening tests for cervical cancer. $N$ Engl J Med, 357, 1579-8.

Mirabello L, Schiffman M, Ghosh A, et al (2013). Elevated methylation of HPV16 DNA is associated with the development of high grade cervical intraepithelial neoplasia. Int J Cancer, 132, 1412-2.

Nicolas Wentzensen N, Sherman M, Schiffman M, Wang S (2008). Utility of methylation markers in cervical cancer early detection: Appraisal of the state-of-the-science. Gynecol Oncol, 112, 293-9.

Ogilvie G, Krajden M, Niekerk D, Martin R, Ehlen T, Ceballos $\mathrm{K}$, et al (2012). Primary cervical cancer screening with HPV testing compared with liquid-based cytology: Results of round 1 of a randomised controlled trial-the HPV FOCAL study. B J Cancer, 107, 1917-4.

Othman N, Hayati Othman N (2014). Detection of human papillomavirus DNA in routine cervical scraping samples: Use for a national cervical cancer screening program in a developing nation. Asian Pac J Cancer Prev, 15, 2245-9.

Patel D, Rozek L, Colacino J, Van Zomeren-Dohm A, Ruffin M, Unger E, et al (2012). Patterns of cellular and HPV 16 methylation as biomarkers for cervical neoplasia. $J$ Virol Methods, 184, 84-2.

Persson M, Elfstrom M, Wendel S, Weiderpass E, Andersson S (2014). Triage of HR-HPV positive women with minor cytological abnormalities: A comparison of mRNA testing, HPV DNA testing, and repeat cytology using a 4-year follow-up of a population-based study. PLOS ONE, 9, 1-8.

Pimple S, Shastri S (2014). Comparative evaluation of human papilloma virus-DNA test verses colposcopy as secondary cervical cancer screening test to triage screen positive women on primary screening by visual inspection with 5\% acetic acid. Ind $J$ Cancer, 51, 117-3.

Rossi P, Benevolo M, Vocaturo A, Caraceni D, Ciccocioppo L, Frega A, et al (2013). Prognostic value of HPV E6/E7 mRNA assay in women with negative colposcopy or CIN1 histology result: A follow-up study. PLOS ONE, 8, 57600.

Schmitt M, Depuydt C, Benoy I, et al (2013). Viral load of highrisk human papillomaviruses as reliable clinical predictor for the presence of cervical lesions. Cancer Epidemiol Biomarkers Prev, 22, 406-4.

Shalini L, James P, Nancy B, et al (2002). Evaluation of human papillomavirus testing in primary screening for cervical abnormalities Comparison of sensitivity, specificity, and frequency of referral. JAMA, 288, 1749-7.

Trimble C, Frazer I (2009). Development of therapeutic HPV vaccines. Lancet Oncol, 10, 975-0.

Vasiljevic N, Scibior $\neg$ Bentkowska D, Brentnall A, Cuzick J, Lorincz A (2014). Credentialing of DNA methylation assays for human genes as diagnostic biomarkers of cervical intraepithelial neoplasia in highrisk HPV positive women. Gynecol Oncol, 132, 709-4.

Wentzensen N, Vinokurova S, Doeberitz M (2004). Systematic review of genomic integration sites of human papillomavirus genomes in epithelial dysplasia and invasive cancer of the female lower genital tract. Cancer Res, 64, 3878-4.

Widschwendter A, Blassnig A, Wiedemair A, et al (2003). Human papillomavirus DNA in sera of cervical cancer patients as tumor marker. Cancer Letters, 202, 231-9.

Woodman C, Collins S, Young L (2007). The natural history of cervical HPV infection: Unresolved issues. Nat Rev Cancer, 7, 11-2.

Xu B, Chotewutmontri S, Wolf S, Klos U, Schmitz M (2013). Multiplex identification of human papillomavirus 16 DNA integration sites in cervical carcinomas. PLOS ONE, 8, e66693. 